\title{
METASTABILITY AND RYDBERG STATES OF TRIATOMIC HYDROGEN
}

\author{
C. BORDAS* and H. HELM
}

Molecular Physics Laboratory, SRI International, 333 Ravenswood Avenue, Menlo Park, CA 94025, USA

${ }^{*}$ LASIM, Université Lyon I, 43 boulevard du 11 novembre 1918, F-69622 Villeurbanne cedex, France

\begin{abstract}
The np,nd and nf Rydberg series of $\mathrm{H}_{3}$ have been studied by one- or twophoton excitation from the lowest metastable state of $\mathrm{H}_{3}: \mathrm{B} 2 \mathrm{p}^{2} \mathrm{~A}_{2}$. The lifetime of the metastable state has been measured and the influence of an external electric field on the Rydberg states has been studied under both aspects of dynamics (field-ionization and fieldinduced predissociation) and structure (Stark effect).
\end{abstract}

\section{1 - Introduction.}

Although the $\mathrm{H}_{3}^{+}$ion, one of the most compact and stable molecular ion, has been among the first species known to mass spectroscopists, it was not until 1968 that the first observation of the neutral triatomic hydrogen species was reported ${ }^{1}$. The repulsive character of the ground state of $\mathrm{H}_{3}$ was the major impediment to the observation of this unstable molecule. Since then, long-lived $\mathrm{H}_{3}$ molecules have been observed in a variety of experiments but the metastability of the B $2 p^{2} A_{2}^{\prime \prime}$ state ${ }^{2}$ in its rotationless level has been understood only during the past decade 3 . Taking advantage of the metastability of this state in a single rotationnal level $(\mathrm{N}=0)$ while all other low-lying states are strongly predissociate, very simple excitation spectra of $\mathrm{H}_{3}$ Rydberg series may be obtained. Owing to the very high stability of the $\mathrm{H}_{3}^{+}$ion, Rydberg states of $\mathrm{H}_{3}$ are tightly bound while embedded in the continuum of the repulsive ground state $\mathrm{X}$ $\left(1 \mathrm{sa}_{1}\right)^{2} 2 \mathrm{p}{ }^{2} \mathrm{E}^{\prime}$ of $\mathrm{H}_{3}$. No repulsive state cross the Rydberg states and thus they do not suffer direct predissociation. On the other hand, penetration effects are weak and electron-core couplings are limited due to the small size of the $\mathrm{H}_{3}^{+}$core.

In our experiments, a $1.5 \mathrm{keV}$ beam of $\mathrm{H}_{3}^{+}$ions is mass selected and neutralized in a Cs charge exchange cell to produce a neutral $\mathrm{H}_{3}$ beam consisting almost completely of molecules in the $\mathrm{B}$ state (more than $80 \%$ in the vibrationless level). The molecular beam interacts with a counterpropagating pulsed laser beam over a region about $120 \mathrm{~cm}$ long. In the central portion of this interaction region, a well defined electric field is applied and the production of excited states is monitored by electric field ionization of the resulting $\mathrm{H}_{3}^{+}$ions. An appropriate gating allows us to record only the ions corresponding to neutral molecules excited in the region where the electric field is well defined. This experimental set-up enables us to characterize the B state which is largely dominant in the beam, and to study Rydberg series by one- or two-photon resonance excitation. 


\section{2 - Metastability of the $B 2 p^{2} A_{2}^{n}$ state.}

Using direct photoionization 4 of the neutral molecules (with a UV photon, $\lambda<338 \mathrm{~nm}$ ) in the absence of any external field and recording the ion signal as a function of the time of flight of the $\mathrm{H}_{3}$ molecules, we can deduce the amount of neutral molecules in the beam as a function of the time elapsed since their formation and then determine directly the lifetime of the metastable state. We find the value of $640_{-100}^{+300} \mathrm{~ns}$, in complete disagreement with the expected value of $88 \mu$ s calculated 5 on the assumption of a purely radiative decay towards the dissociative $2 \mathrm{~s}^{2} \mathrm{~A}_{2}$ state. We attribute the faster decay channel to weakly allowed radiative transitions between the metastable state and the degenerate mode excited repulsive ground state $\mathrm{H}_{3}$, as well as to predissociation induced by spin-orbit coupling. Numerical estimations are not available for the radiative process while one can estimate that the spin-orbit coupling may account only for $10 \%$ of the fast observed decay rate.

\section{3 - Field free Rydberg states spectroscopy.}

Using the $B$ state which has a strong $2 p$ character as initial state, ns and nd Rydberg series may be excited by one photon transitions while the excitation of $\mathrm{np}$ or $\mathrm{nf}$ series requires a two photon excitation via a ns or nd state respectively. Figure 1 shows the $n$, nd and $n f$ series converging to the first ortho-level of the $\mathrm{H}_{3}^{+}$ion $\left(\mathrm{N}^{+}=1\right)$. The ns series has not been observed for high $\mathrm{n}$ values because it is about 40 times less intense than the nd series and it is probably predissociate.

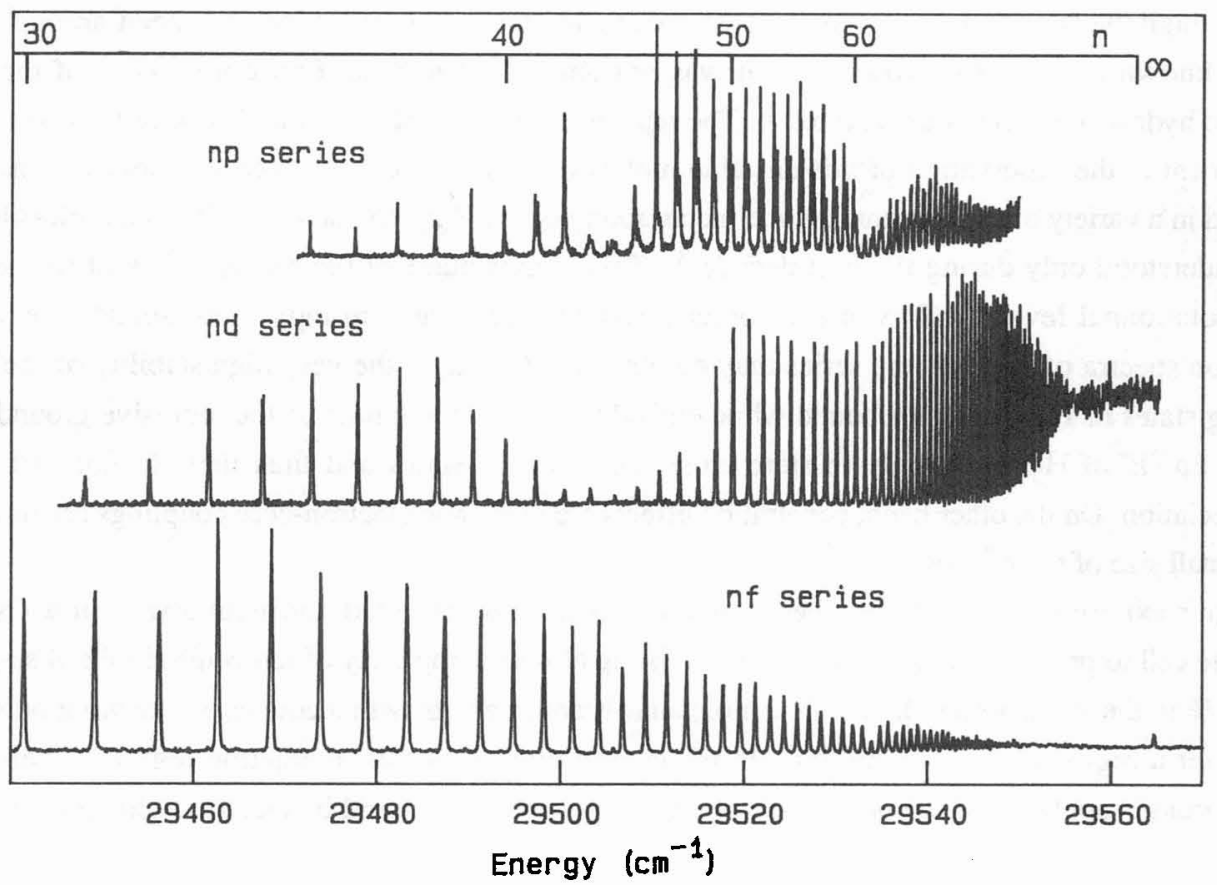

Figure 1 : from top to bottom, $n p$, nd and $n f$ Rydberg series of $\mathrm{H}_{3}$ converging toward the lowest ortho-level $(N+=1)$ of the ion. 
np series : these series have been excited ${ }^{6}$ using optical-optical double resonance via the $3 \mathrm{~s}^{2} \mathrm{~A}_{1}$ $\left(\mathrm{N}=1, \mathrm{v}_{1}=0, \mathrm{v}_{2}=0\right)$ intermediate level. Bound states below the $\mathrm{N}^{+}=1$ level of the ion detected by fieldionization as well as autoionizing states between the $\mathrm{N}^{+}=1$ and $\mathrm{N}^{+}=3$ levels of the ion have been characterized using a two-channel rotationnal quantum defect theory. MQDT allows us to analyse simply the mixing of the $n p^{2} A_{2}^{\prime}$ and $n p{ }^{2} E^{\prime}$ series in Hund's case (b) that correspond to $n p N^{+}=1$ and $n p N^{+}=3$ series in Hund's case (d). Deviations from the calculation appear when vibrational interactions give rise to accidental predissociation below threshold or autoionizing interlopers above threshold. As opposed to the other Rydberg series of $\mathrm{H}_{3}$ which show almost now electron-core interactions, the np series of $\mathrm{H}_{3}$ provide a complete panorama of channel interactions in a simple polyatomic molecule: rotationnal and vibrationnal interactions and autoionizations with rich Fano-profiles structure above threshold as well as indirect predissociation induced by high $\mathrm{v}-$ low $\mathrm{n}$ interlopers.

nd series : very simple nd series converging toward the $\mathrm{N}^{+}=1$ level of the $\mathrm{H}_{3}^{+}$ion has been observed 7 for $n$ values ranging from $n=25$ to $n=125$. A precise value of the ionization potential of $H_{3}$ has been extrapolated from this series: $29562.58 \mathrm{~cm}^{-1}$ above the metastable level. The nd series is almost free of core interactions (very small quantum defect of 0.02 ) and it has a quasi atomic aspect except the very strong intensity window observed in the region $n=40$ to 48 (Fig. 1) and related to predissociation induced by a highly vibrationnaly excited interloper.

nf series : from the initial B state, two $3 \mathrm{~d}$ levels are accessible by one photon excitation. The $3 \mathrm{~d}$ 2E" and the $3 \mathrm{~d}^{2} \mathrm{~A}_{1}$ states $(\mathrm{N}=1)$ which are used as initial levels to excite the $\mathrm{nf}$ series. The observed spectra 8 show that: (i) the $\mathrm{nf}$ series is even less perturbed than the nd series by electron-core interactions (quantum defect $\approx 0.01$ and almost no observed predissociation) (ii) the $3 \mathrm{~d}^{2} \mathrm{E}^{\prime \prime}$ and the $3 \mathrm{~d}^{2} \mathrm{~A}_{1}$ states are in fact almost pure case (d) states and the notation $3 \mathrm{dN}^{+}=1$ and $3 \mathrm{dN}^{+}=3$ respectively should be preferred instead. This is emphasized by the nf spectrum of figure 1 recorded with the intermediate state " $3 \mathrm{~d}{ }^{2} \mathrm{E}$ " " where only $\mathrm{N}^{+}=1$ states are seen

\section{4 - Electric field effects :}

At moderate field strength, predissociation affects selected $\mathrm{p}$ and $\mathbf{d}$ Rydberg states of high $\mathbf{n}$ values. Predissociation causes a decrease in the observed intensities (no $\mathrm{H}_{3}^{+}$ions are produced), this is clearly visible for instance in the region around $n=44$ of the nd spectrum (Fig. 1). The field-induced predissociation is strikingly demonstrated in the nd series at $n=61$ (see Fig. 2): an electric field as low as $0.4 \mathrm{~V} / \mathrm{cm}$ is responsible for the complete disappearance of the $\mathrm{n}=61 \mathrm{line}$, which, according to our experimental conditions, corresponds to a lifetime relative to predissociation of about $300 \mathrm{~ns}$. We have shown 9 that a relatively weak coupling with a vibrationnaly excited state of low principal quantum number that exhibits significant coupling to the repulsive ground state may be responsible for this effect. Quantitative interpretation of the field-induced predissociation processes that are crucial for dissociative recombination of $\mathrm{H}_{3}^{+}$is presently under way. At higher field strength, partly resolved Stark spectra have been obtained in the intermediate and strong ( $\mathrm{n}$-mixing) field regime ${ }^{10}$. We have developed a perturbative model of the Stark effect of $\mathrm{H}_{3}$ which takes into account the $\mathrm{n}$-mixing and the coupling of the different angular momenta. This treatment represents the first complete calculation of the Stark effect in molecular 


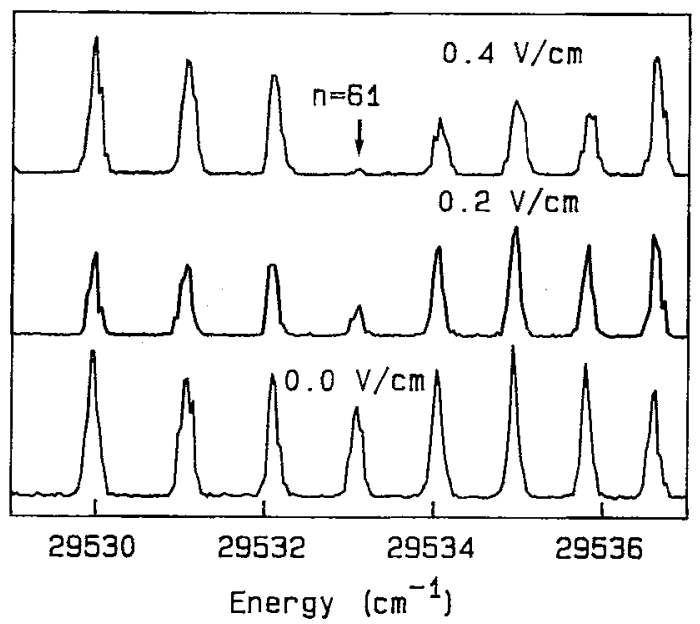

Fig 2 : nd Rydberg series in the vicinity of $n=61$ for low field values.

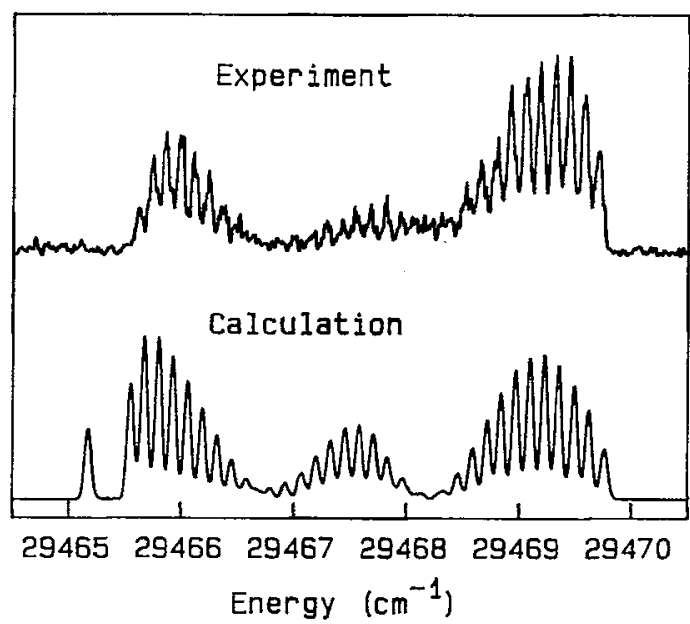

Fig. 3 : experimental and calculated Stark manifold for $n=34, M_{N}=0$ and $F=30 \mathrm{~V} / \mathrm{cm}$.

Rydberg states. The good knowledge of all the quantum defects combined with the single $\mathrm{N}^{+}=1$ level of the ionic core involved in the problem has allowed this very succesful treatment. Fig. 3 shows the excellent agreement between experiment and calculation for the $n=34, M_{N}=0$ manifold in a field $F=30 \mathrm{~V} / \mathrm{cm}$. This perturbative model accounts very well for the observed spectra except for the intensity windows attributed to accidental predissociation.

\section{Conclusion :}

Though relatively recent, the study of the Rydberg series of $\mathrm{H}_{3}$ is nowadays one of the most complete in the area of molecular Rydberg states. The very good agreement between ab-initio calculations and experimental results usually observed will probably allow in the future the complete understanding of the complex interactions leading to the numerous predissociations observed below the ionization threshold.

1/F. M. Devienne, C. R. Acad. Sci., Ser. B 267, 1279 (1968)

2 / G. Herzberg, J. Chem. Phys. 70, 4806 (1979)

3 / G. I. Gellene and R. F. Porter, J. Chem. Phys. 79, 5975 (1983)

4 / C. Bordas, P. C. Cosby and H. Helm, J. Chem. Phys. 93, 6303 (1990)

5 / H. F. King and K. Morokuma, J. Chem. Phys. 71, 3213 (1979)

6 / C. Bordas, L. J. Lembo and H. Helm, Phys. Rev. A 44 (1991)

7/ H. Helm, Phys. Rev. A 38, 3425 (1988)

8 / L. J. Lembo, M. C. Bordas and H. Helm, Phys. Rev. A 42, 6660 (1990)

9 / C. Bordas and H. Helm, Phys. Rev. A 43, 3645 (1991)

$10 /$ C. Bordas and H. Helm, submitted to Phys. Rev. A 